ARTIFICIAL SATELLITES, Vol. 50, No. 2 - 2015

DOI: 10.1515/arsa-2015-0005

\title{
GNSS-WARP SOFTWARE FOR REAL-TIME PRECISE POINT POSITIONING
}

\author{
Hadaś Tomasz \\ Wroclaw University of Environmental and Life Sciences, \\ Institute of Geodesy and Geoinformatics \\ ul. Grunwaldzka 53, 50-357 Wrocław \\ e-mail: tomasz.hadas@up.wroc.pl
}

\begin{abstract}
On April 1, 2013 IGS launched the real-time service providing products for Precise Point Positioning (PPP). The availability of real-time makes PPP a very powerful technique to process GNSS signals in real-time and opens a new PPP applications opportunities. There are still, however, some limitations of PPP, especially in the kinematic mode. A significant change in satellite geometry is required to efficiently de-correlate troposphere delay, receiver clock offset, and receiver height. In order to challenge PPP limitations, the GNSS-WARP (Wroclaw Algorithms for Real-time Positioning) software has been developed from scratch at Wroclaw University of Environmental and Life Science in Poland. This paper presents the GNSS-WARP software itself and some results of GNSS data analysis using PPP and PPP-RTK (Real-Time Kinematic) technique. The results of static and kinematic processing in GPS only and GPS + GLONASS mode with final and real-time products are presented. Software performance validation in postprocessing mode confirmed that the software can be considered as a state-ofthe-art software and used for further studies on PPP algorithm development. The real-time positioning test made it possible to assess the quality of real-time coordinates, which is a few millimeters for North, East, Up in static mode, a below decimeter in kinematic mode. The accuracy and precision of height estimates in kinematic mode were improved by constraining the solution with an external, near real-time troposphere model. The software also allows estimation of real-time ZTD, however, the obtained precision of $11.2 \mathrm{~mm}$ means that further improvements in the software, real-time products or processing strategy are required.
\end{abstract}

Keywords: PPP, real-time positioning, IGS RTS, GNSS software, troposphere 


\section{INTRODUCTION}

Precise Point Positioning (PPP) is a positioning technique that uses a single GNSS (Global Navigation Satellite System) receiver that requires external information from analysis of the global GNSS permanent network. The original PPP concept was to introduce precise orbits and satellite clocks into the equation system as known parameters (Zumberge et al 1997). Resulting coordinates are strictly connected with the orbital reference frame, which forces the inclusion of site correction models (solid earth tides, polar tides, ocean loading) (Héroux et al 2000). The data is processed in a zero-differences level, so for precise applications it is necessary to consider satellite and receiver effects models: satellite antenna offsets, phase wind-up, receiver antenna offset and variation (Kouba, 2009). It is also necessary to deal with troposphere and ionosphere delays. The first order ionosphere delay can be removed when processing dual frequency GNSS data which allows computing ionosphere-free linear combination L3 of code and carrier measurements. The tropospheric delays remain as estimated parameters that are represented in the equation system by zenith total delay (ZTD) and calculated into delays along the receiversatellite paths using external mapping functions.

This technique is commonly used in post-processing mode and gives results comparable to the relative positioning after processing a few hours of data. In this case, PPP users take advantage of precise GNSS products provided, for example, by the International GNSS Service (IGS). The products are derived with some delay, reaching up to 18 days for final products (Dow et al 2009). In order to satisfy real-time users, on April 1, 2013 IGS launched real-time service (RTS), currently providing official GPS and unofficial GLONASS products including orbits, clocks, and code biases. The RTS goal is to provide a reliable real-time GNSS combination product free of outages and outliers (Mervart and Weber 2011). The detailed description and verification of the products was presented by Hadas and Bosy (2014), where the high quality and availability of RTS were proved. The RTS is constantly monitored by the real-time analysis center coordinator, by comparing the official RTS products daily with IGS rapid solution. The results are presented in the IGS RTS webpage (http://rts.igs.org/).

The availability of real-time makes PPP a very powerful technique to process GNSS signals in real-time and opens new PPP application opportunities e.g. ocean buoy positioning for tsunami detection (Kanzaki et al 2011), deformation monitoring (Chen et al 2009), seismic-related displacements detection (Hefty and Gerhatova, 2012). A shortcoming of this technique, however, is the time required for the solution to converge. This is caused mainly by two factors. The first factor is the use of ionosphere-free linear combination that loses the integer nature of ambiguities. This limits the application of ambiguity fixing techniques unless the raw signals are processed; however, in this case, one has to apply precise ionospheric correction (Juan et al 2012, Zhang et al 2013) or introduce regional satellite clocks (Laurichesse 2011). The second factor is the high correlation among the estimated parameters: troposphere delay, receiver clock offset, and receiver height. To efficiently de-correlate these parameters, a significant change in satellite geometry is required. Alternatively, one can introduce the external high-quality regional troposphere delay model as a priori model, which has a positive effect on precision, accuracy, and convergence time (Jensen and Ovstedal 2008, Hadas et al 2013).

The limitations of PPP can be overcome with data from GBAS (Ground Based Augmentation System) networks, that can derive many GNSS error corrections in real-time. Such a concept of combining the PPP with RTK (Real Time Kinematics) networking is called PPP-RTK (Wübbena 
et al 2005). This technique has the potential of providing rapid convergence within a few centimeters accuracy (Geng et al 2010, Li et al 2011).

In order to challenge some PPP limitations, to develop and verify the original PPP and PPPRTK algorithms, the state-of-the-art software GNSS-WARP (Wroclaw Algorithms for Real-time Positioning) has been developed from scratch at Wroclaw Univeristy of Environmental and Life Science (WUELS), Poland. The software is capable of processing static and kinematic multiGNSS data in real-time and post-processing mode and takes advantage of IGS RTS products as well as final IGS products. Various global troposphere models and mapping functions are included in the software, but regional models are also supported, including the near real-time (NRT) ZTD model developed at WUELS (Bosy et al 2010, Bosy et al 2012).

This article presents the GNSS-WARP software itself and some of the results of GNSS data analysis using PPP and PPP-RTK technique. Section 2 describes the GNSS-WARP architecture, implemented PPP functional adjustment model, data processing scheme, and software functionality. Section 3 provides confirmation that the developed GNSS-WARP is state-of-the-art software. In this part, the results of kinematic and static data processing in postprocessing mode are presented and compared with the reference solution.

The following sections will discuss real-time applications. Section 4 will cover GPS and GPS+GLONASS static and kinematic positioning using IGS real-time products. Then, in section 5 , the original method of constraining the kinematic positioning with regional troposphere model is presented and some exemplary results demonstrate the advantage of PPP-RTK positioning. The preliminary results of real-time ZTD estimation for selected European Permanent Network (EPN) stations over area of Poland are presented in section 6 . The real-time estimates are compared with well-established near real-time network solution. Finally, section 7 summarizes and concludes the information presented in this article and includes further indications of future software development plans and future research objectives.

\section{GNSS-WARP SOFTWARE}

The GNSS-WARP software is implemented in Matlab, but is not stand-alone software. For realtime processing it requires BKG Ntrip Client (BNC) software provided by BKG (2013) to handle Networked Transport of RTCM via Internet Protocol (NTRIP) for streaming GNSS and differential correction data over the Internet (Weber et al. 2005). The observables from the GNSS receiver and broadcast messages are decoded from RTCM 3.x format and RTS correction streams are decoded from a RTCM state space representation (SSR) standard (Wübbena et al. 2005). Then the observables, broadcast messages, and SSR corrections are provided in text-formatted messages by TCP/IP. This protocol is supported by Matlab Instrument Control Toolbox so that the data can be constantly and immediately imported into Matlab workspace as structures and variables. All the remaining data, e.g. troposphere delays and mapping functions, is imported from FTP servers in original formats and decoded with procedures developed in Matlab. The real-time data flow in GNSS-WARP is presented in Figure 1. The GNSS-WARP is also capable of processing data in postprocessing mode. This mode was originally developed to debug realtime algorithms and to compare the different processing strategies. In this case BNC is not required and the data feed algorithms are implemented as Matlab functions, providing the data in exactly the same structures and variables as real-time mode.. 


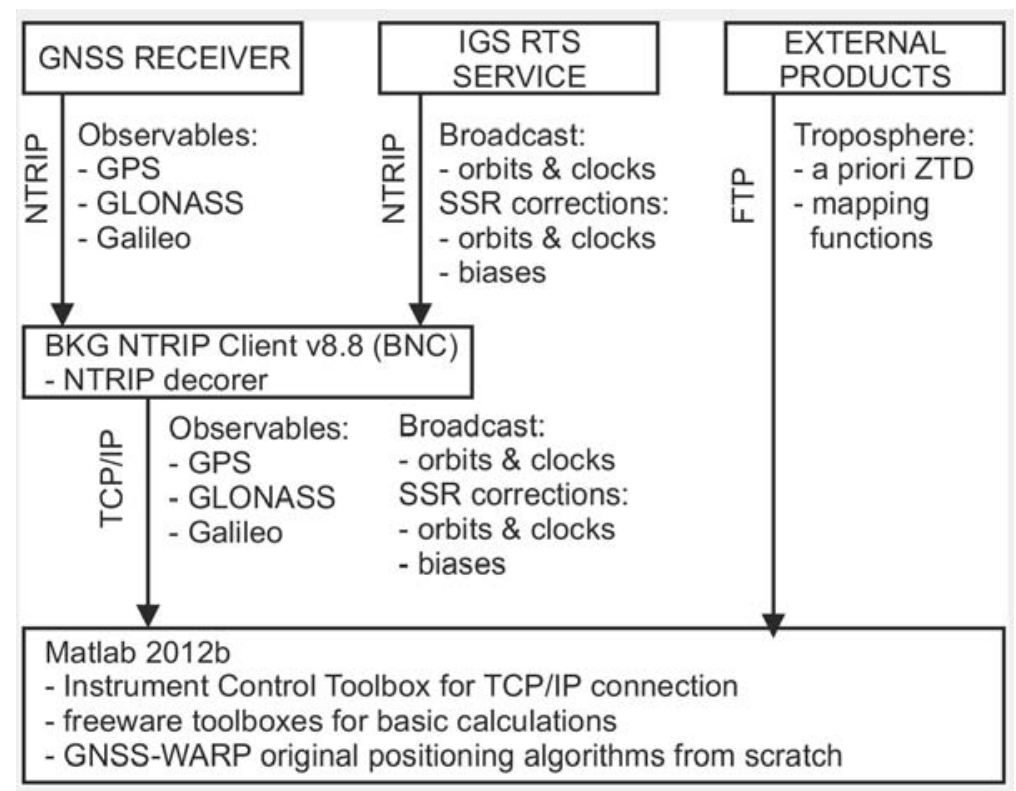

Fig. 1. Data sources and transmission protocols in GNSS-WARP software

The software is highly configurable, capable of processing static and kinematic data. Currently it supports GPS and GLONASS, but the algorithms for Galileo are also implemented, and the system will be included into processing as soon as the real-time streams or precise products become available. The software makes it possible to save the calculated variables and estimated parameters into log files, import them, and provide detailed analysis and statistics of the results. The skyplot, receiver $2 \mathrm{D}$ and $3 \mathrm{D}$ position, and time series of parameter values (e.g. DOP, number of satellites, coordinate errors, etc.) are presented in real-time plots that dynamically adjust to the value range and data time window.

The data processing is done on an epoch-by-epoch basis, combining the functional model for pseudorange

$$
P_{i f}^{i}=\rho^{i}-\frac{\left(X^{i}-X_{r}\right)}{\rho} \delta X_{r}-\frac{\left(Y^{i}-Y_{r}\right)}{\rho} \delta Y_{r}-\frac{\left(Z^{i}-Z_{r}\right)}{\rho} \delta Z_{r}+c \cdot \delta t_{r}-c \cdot \delta t^{i}+Z T D \cdot M F^{i}+\Delta_{P}^{i}
$$

and carrier-phase measurements:

$$
\Phi_{i f}^{i}=\rho^{i}-\frac{\left(X^{i}-X_{r}\right)}{\rho} \delta X_{r}-\frac{\left(Y^{i}-Y_{r}\right)}{\rho} \delta Y_{r}-\frac{\left(Z^{i}-Z_{r}\right)}{\rho} \delta Z_{r}+c \cdot \delta t_{r}-c \cdot \delta t^{i}+Z T D \cdot M F^{i}+\lambda_{i f} \cdot N_{i f}^{i}+\Delta_{\Phi}^{i}
$$

where:

- $\quad i$ is the satellite index;

- $\quad P_{i f}^{i}$ and $\Phi_{i f}^{i}$ are iono-free combination of pseudorange and carrier-phase measurements respectively;

- $\quad \rho^{i}$ is the geometric distance between receiver and satellite;

- $\quad X^{i}, Y^{i}, Z^{i}$ are the satellite precise coordinates;

- $X_{r}, Y_{r}, Z_{r}$ are the receiver a priori coordinates;

- $\delta X_{r}, \delta Y_{r}, \delta Z_{r}$ are the updates to the receiver a priori coordinates;

- $\delta t_{r}$ and $\delta t^{i}$ are the receiver and satellite clock corrections respectively; 
- $c$ is the speed of the light;

- ZTD is the zenith troposphere delay;

- $M F^{i}$ is the troposphere mapping function;

- $\lambda_{\text {if }}$ is the iono-free wavelength;

- $\quad N_{i f}^{i}$ is the iono-free ambiguity parameter;

- $\Delta_{P}^{i}$ and $\Delta_{\Phi}^{i}$ are satellite, receiver and site displacements effects corrections for pseudorange and carrier-phase measurements respectively.

First, the filter for receiver position is initialized with an a priori coordinates, while the filters for receiver clock and iono-free ambiguity parameters are set to 0 . The variances in initial covariance matrix are defined with an a priori sigma of respective parameters, and covariances are set to 0.Then, the Single Point Positioning (SPP) using raw pseudoranges, broadcast orbits and clocks is performed for clock synchronization, that is to obtain the accurate epoch of signal emission from each satellite. This step is required to calculate satellite precise orbits and clocks. Depending on the processing configuration, the a priori receiver position comes from the last epoch result or code-based positioning. The a priori receiver position is used to obtain a priori zenith troposphere delay and mapping functions from a chosen model, e.g. UNB3 (Leandro et al. 2006), GPT2 (Lagler et al. 2013), VMF (Böhm et al. 2009). The variance of troposphere delay is initialized in covariance matrix based on the accuracy of chosen model. The a priori receiver position is also used to calculate receiver, satellite and site displacement effects which include receiver antenna phase center (APC) offset and variation, phase wind-up, relativistic effect, satellite APC offset, differential code biases, solid earth tides, ocean tide loading, and atmosphere pressure loading. Then the ionosphere-free combination of pseudorange and carrier-phase measurements are calculated

$$
\begin{aligned}
P_{i f}^{i} & =\frac{f_{1}^{2} \cdot P_{1}^{i}-f_{2}^{2} \cdot P_{2}^{i}}{f_{1}^{2}-f_{2}^{2}} \\
\Phi_{i f}^{i} & =\frac{f_{1}^{2} \cdot \Phi_{1}^{i}-f_{2}^{2} \cdot \Phi_{2}^{i}}{f_{1}^{2}-f_{2}^{2}}
\end{aligned}
$$

where $P_{1}^{i}$ and $P_{2}^{i}$ are pseudorange measurements to the $i$-th satellite on two frequencies, $\Phi_{1}^{i}$ and $\Phi_{2}^{i}$ are carrier-phase measurements to the $i$-th satellite on two frequencies, $f_{1}$ and $f_{2}$ are the frequencies of both signals used. The update vector $\delta$ is computed with a modified least square adjustment:

$$
\delta=\left(A^{T} P A+C_{X}^{-1}\right)^{-1} A^{T} P l
$$

where $A, P$ and $C_{X}$ are the design, weight and covariance matrixes respectively, $l$ is the misclosure vector (Leandro et al. 2007). If outliers are detected with the local test (adjusted measurement correction larger the $3 \sigma$ confidence limit), the adjustment is recalculated until the global test (Chi-squared) is reached. Finally, if the valid solution is obtained, the receiver position, ambiguity and troposphere delay filter are overridden. The covariance matrix is updated

$$
C_{X}{ }^{\prime}=\left(A^{T} P A+C_{X}{ }^{-1}\right)^{-1}+C_{\text {noise }}
$$

where $C_{\text {noise }}$ represents the process noise, thus allowing to treat parameters as constant, stochastic or white noise. For constant parameters (static receiver coordinates, phase ambiguities when until 
cycle slip occurs), no noise is introduced into $C_{X}{ }^{\prime}$ - the respective values in $C_{\text {noise }}$ remains 0 . For stochastic parameters (troposphere delay) the $C_{\text {noise }}$ allows to increase the variance of parameter by a predefined value of random walk step. For white noise parameters (receiver kinematic coordinates, receiver clock offset, phase ambiguity in case of cycle slip) the variances in $C_{X}{ }^{\prime}$ are reset to initial values, and covariances should be set to 0 .

\section{SOFTWARE PERFORMANCE VALIDATION}

To validate the performance of GNSS-WARP software, the postprocessing functions were developed. In the postprocessing mode the software is capable of processing GPS and GLONASS data from RINEX files, importing precise ephemeris from SP3 files and precise clocks from CLK files. Once the data is imported into structures at the beginning of the processing, the workspace variables are updated epoch by epoch, thus simulating the real-time processing. Because the same set of data can be processed with various settings and using different processing strategies, it is possible to confirm the correct operation of implemented algorithms and assess the impact of original developments.

Initially, the GNSS-WARP software was used to process one week of daily RINEX files from 20 evenly distributed IGS core stations (Figure 2a). The IGS stations were chosen because of the availability of the official IGb08 solution. The IGb08 coordinates from the latest release were used as a reference for further comparisons. Using the IGS final orbits and clocks, the processing was performed in static and kinematic modes. In kinematic positioning, the part of the covariance matrix that corresponds to station coordinates was reset every epoch, while in static mode it was updated by the results from the previous epoch, according to the equation (6). In both cases, the data sampling was set to 30 seconds according to the interval of IGS final high-rate clocks. The Vienna Mapping Function service was used to provide troposphere mapping functions and a priori troposphere delay. The elevation cut-off angle was set to 5 degrees and the ambiguities remain as float values.

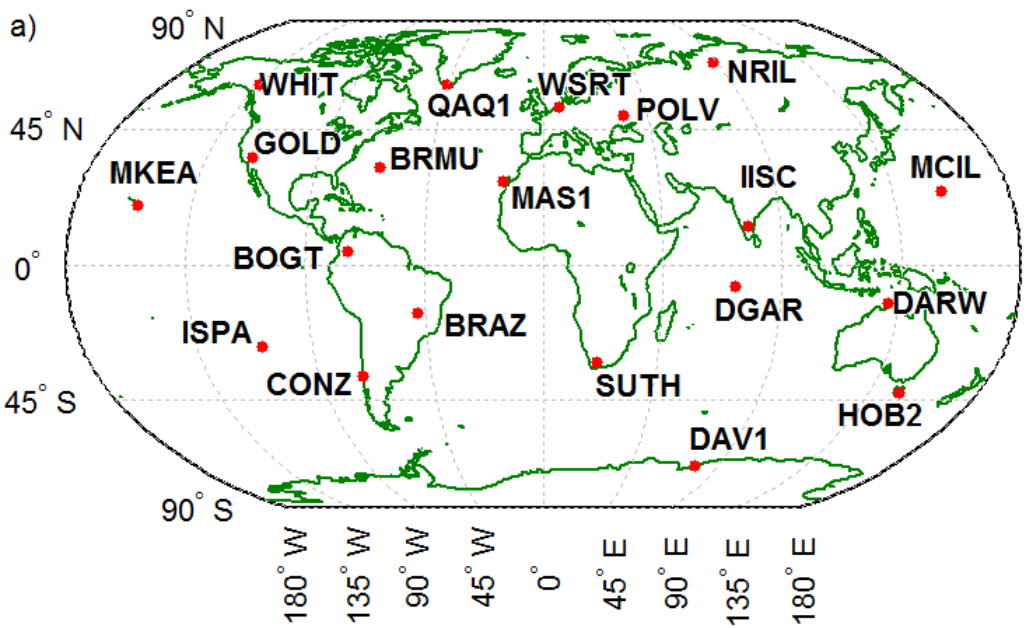

b)

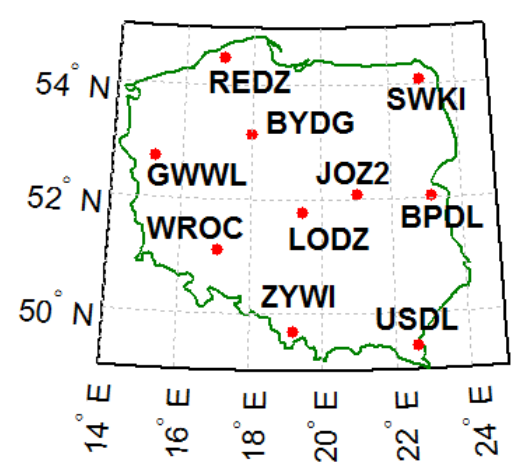

Fig. 2. Location of permanent stations used in the experiments: a) 20 selected IGS core stations b) 10 selected EPN stations in Poland 
The exemplary results of 6-hour GPS data processing from station WROC are presented in Figure 3 for static and kinematic modes. In static mode, it takes about half of an hour for the solution to converge below $1 \mathrm{dm}$ accuracy for each coordinate component when considering the difference between estimated and reference IGb08 coordinates. After 6 hours of data processing, the residuals from known station coordinates differ less than $2 \mathrm{~cm}$. At the same time, the estimated error is around $1 \mathrm{~cm}$ and $3 \mathrm{~mm}$ after processing 1 and 6 hours of data respectively. In kinematic mode, the solution requires about 45 minutes to converge, but in this case the residuals are around $10 \mathrm{~cm}$ in horizontal and vertical component, and remains below this level for the entire remaining time. The estimated error is usually below $3 \mathrm{~cm}$ for horizontal components and 4 $\mathrm{cm}$ for vertical component, but reaches up to $4 \mathrm{~cm}$ and $7 \mathrm{~cm}$ for horizontal and vertical component respectively.
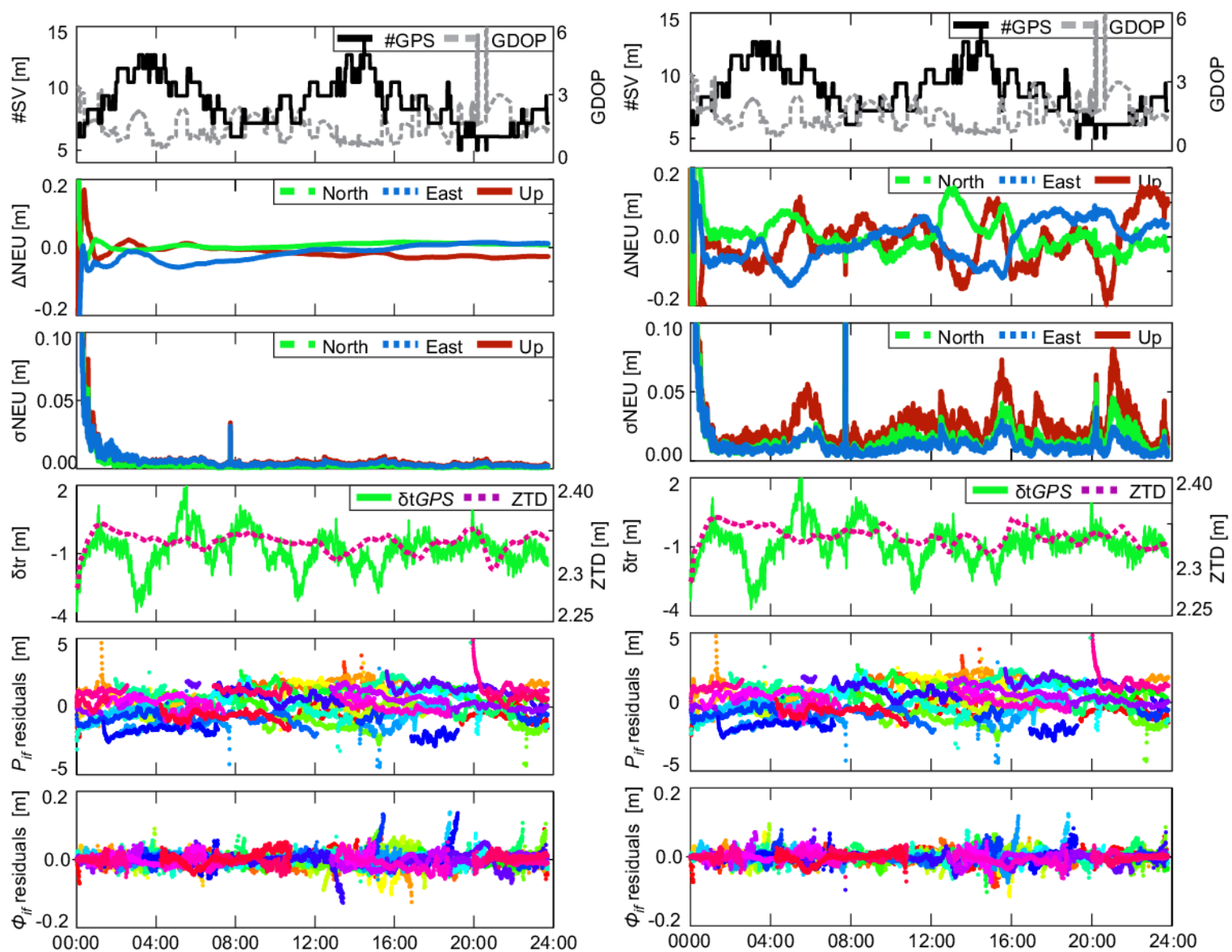

Fig. 3. Time series of estimated parameters in GPS only static (left) and kinematic (right) mode for station WROC, DOY 053, 2014, 00:00 - 06:00

The overall statistics including median, standard deviation, and range of coordinate residuals for 20 IGS core stations over a selected one week are presented in Figure 4 for static (left) and kinematic (right) mode. The residuals from first hour of data processing were removed, assuming that this time was required for the solution to converge. In static mode, the mean value for all residuals was $-0.2 \mathrm{~mm}$ for North and $+1.2 \mathrm{~mm}$ for East, with standard deviations of $1.5 \mathrm{~mm}$ and $2.1 \mathrm{~mm}$ respectively. For the Up component the mean value was $+2.4 \mathrm{~mm}$ with standard deviation of $7.3 \mathrm{~mm}$. In $99.9 \%$, the residuals did not exceed $2 \mathrm{~cm}, 4 \mathrm{~cm}$, and $6 \mathrm{~cm}$ for North, 
East, and Up components respectively. The results confirmed that the solution was free of systematic errors, providing coordinates that were stable over time.

In kinematic mode, the mean values for horizontal coordinate residuals were $-0.9 \mathrm{~mm}$ for North and $+3.9 \mathrm{~mm}$ for East, with standard deviations of $29 \mathrm{~mm}$ and $31 \mathrm{~mm}$, respectively. As for the Up component the values were $-6.4 \mathrm{~mm}$ and $7.2 \mathrm{~mm}$ for mean and standard deviation, respectively. The residuals reached $20 \mathrm{~cm}$ and $39 \mathrm{~cm}$ for horizontal and vertical components respectively, although for $95 \%$ of results they did not exceed $10 \mathrm{~cm}$ in horizontal and $15 \mathrm{~cm}$ in vertical component. Although the results in kinematic mode were significantly worse than in static mode, for autonomous kinematic processing the accuracy and precision at $1 \mathrm{dm}$ level should be considered as satisfactory.

The precise and accurate results obtained with final products mean that all the necessary models are properly implemented in GNSS-WARP software, data handling and processing is correct. The software can be considered as the state-of-the-art software and used for further studies on PPP algorithm development.

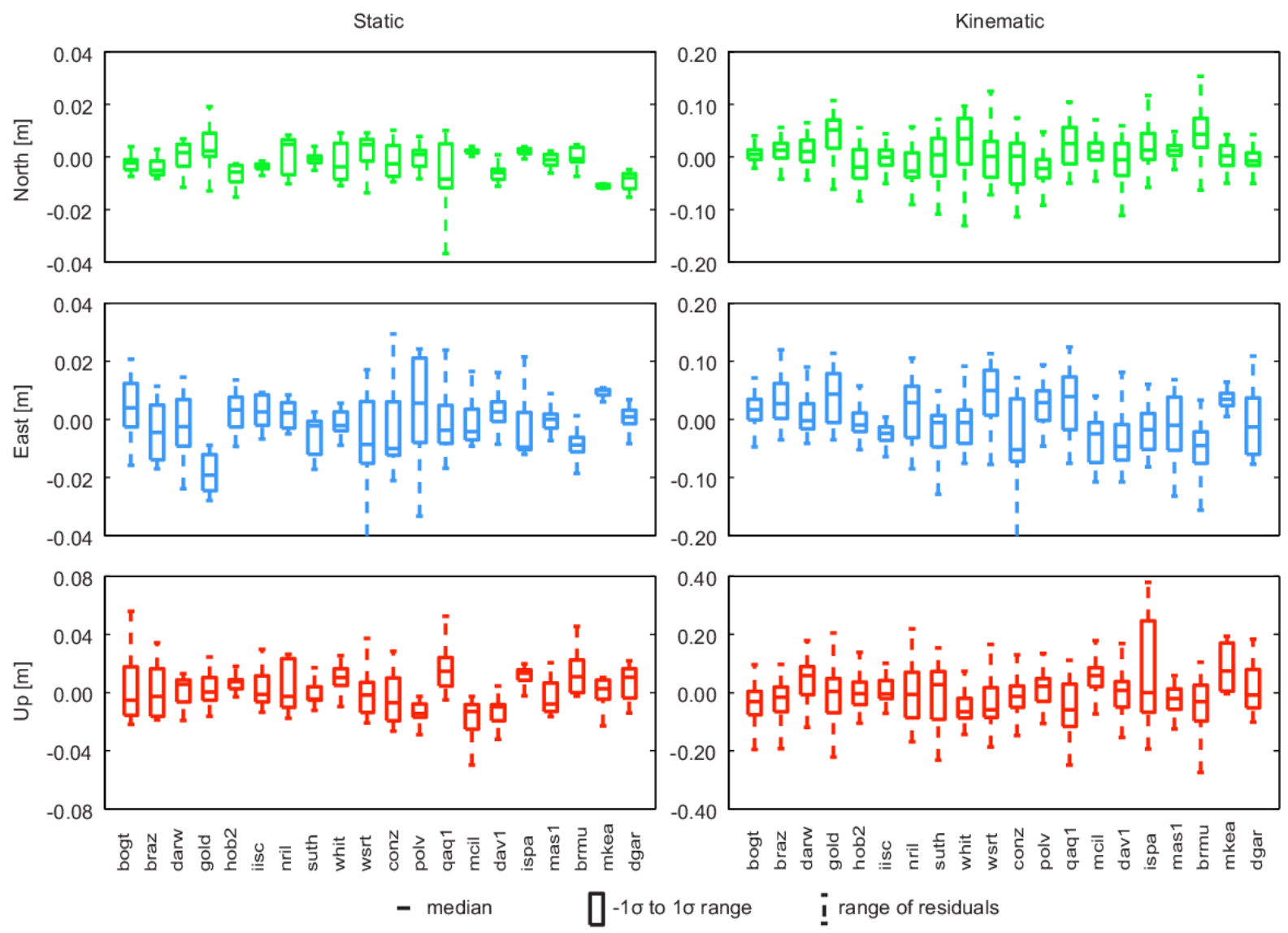

Fig. 4. Statistics of coordinate residuals after 1 hour of convergence time, using final IGS products. 


\section{REAL TIME POSITIONING}

GNSS-WARP software was originally developed as a real-time positioning software. Depending on software configuration, it may be used for different applications. In static mode, it can be used to monitor the stability of control points, e.g. in monitoring of civil engineering objects. The kinematic mode is designed for navigation and positioning under dynamic conditions. The use of real-time positioning software in a specific application requires knowledge of the reliability of provided results, in particular the quality of estimated position.

The software takes advantage of IGS real-time products. IGS RTS was officially launched on April 1, 2013, providing two official streams for GPS. There is also one stream for combined GPS and GLONASS, but this stream is unofficial because the products for GLONASS are still not as accurate as GPS products. For this reason, independent tests for GPS only and GPS+GLONASS were performed, to identify the potential use of the software in real-time applications.

For 10 Polish EPN stations (Figure 2b), one day of $1 \mathrm{~Hz}$ real-time data was processed in 4 configurations simultaneously: static GPS only, static GPS+GLONASS, kinematic GPS only, and kinematic GPS+GLONASS. Due to the limited processing capacity, each station was processed on a different day, starting from DOY 106 up to DOY 115, 2014. The real-time coordinates were compared with known IGb08 station coordinates. The results obtained for different stations and days were very similar to each other and are described in common in Tables 1 and 2. The analyses concern only residuals obtained after the solution converged below centimeter level for static mode and decimeter level for kinematic mode.

Table 1. Mean value of residuals in real-time positioning [m]

\begin{tabular}{|l|r|r|r|r|}
\hline & \multicolumn{2}{|c|}{ Static } & \multicolumn{2}{c|}{ Kinematic } \\
\hline & GPS only & GPS+GLO & GPS only & GPS+GLO \\
\hline North & 0.005 & 0.025 & 0.007 & 0.015 \\
\hline East & 0.007 & 0.012 & 0.004 & 0.004 \\
\hline Up & 0.001 & -0.033 & 0.057 & -0.031 \\
\hline
\end{tabular}

Table 2. Standard deviation of residuals in real-time positioning [m]

\begin{tabular}{|l|r|r|r|r|}
\hline & \multicolumn{2}{|c|}{ Static } & \multicolumn{2}{c|}{ Kinematic } \\
\hline & GPS only & GPS+GLO & GPS only & GPS+GLO \\
\hline North & 0.002 & 0.013 & 0.030 & 0.035 \\
\hline East & 0.006 & 0.018 & 0.027 & 0.032 \\
\hline Up & 0.006 & 0.011 & 0.120 & 0.092 \\
\hline
\end{tabular}

For static positioning the horizontal coordinates were slightly biased, especially if GLONASS satellites were included in the processing. Standard deviations of residuals for each coordinate component were significantly smaller when GPS only processing was performed. At the same time, the estimated error for each component in both solutions were below a few millimeters. This confirmed, that the quality of GLONASS products were not yet as high as for GPS products. 
For static positioning the inclusion of GLONASS data provided significantly worse results. For GPS only solution, the solutions converged below $1 \mathrm{~cm}$ accuracy after about 2 hours, but for GPS+GLONASS solution the convergence time varies from 1 to 6 hours.

In kinematic mode, the quality of the horizontal coordinates obtained with GPS only and GPS + GLONASS solutions was very similar. The difference was noticed for the Up component, where the inclusion of GLONASS data resulted in smaller bias and standard deviation. It also contributed to a reduction of number and size of extreme residuals for Up component. The average time required for the solution to converge into a decimeter level was 1 hour for GPS only mode and 15 minutes for GPS + GLONASS. All the facts mentioned above speak in favor of GPS + GLONASS solution in kinematic positioning, even though the GLONASS products are still unofficial.
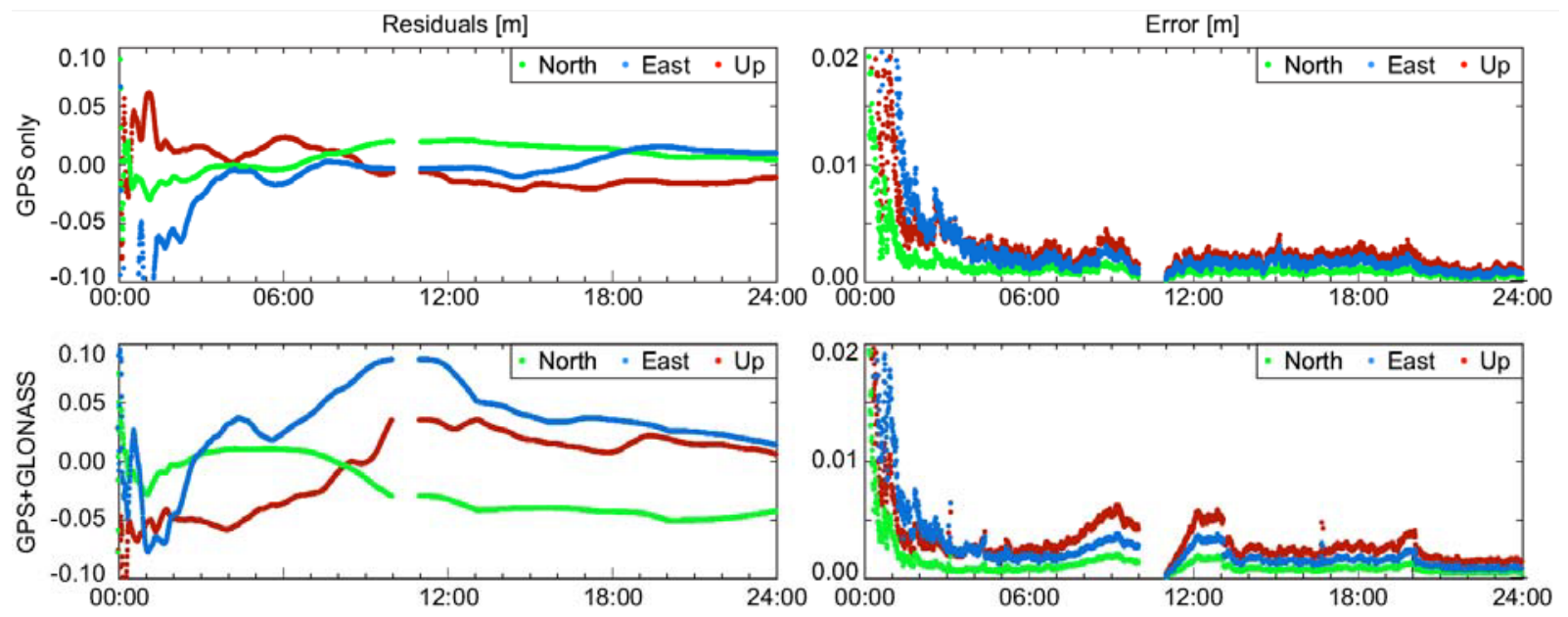

Fig. 5. Time series of residuals and estimated errors for GPS only with IGS01 stream (top) and GPS+GLONASS with IGS03 stream (bottom) real-time positioning in static mode for station WROC, DOY 114, 2014
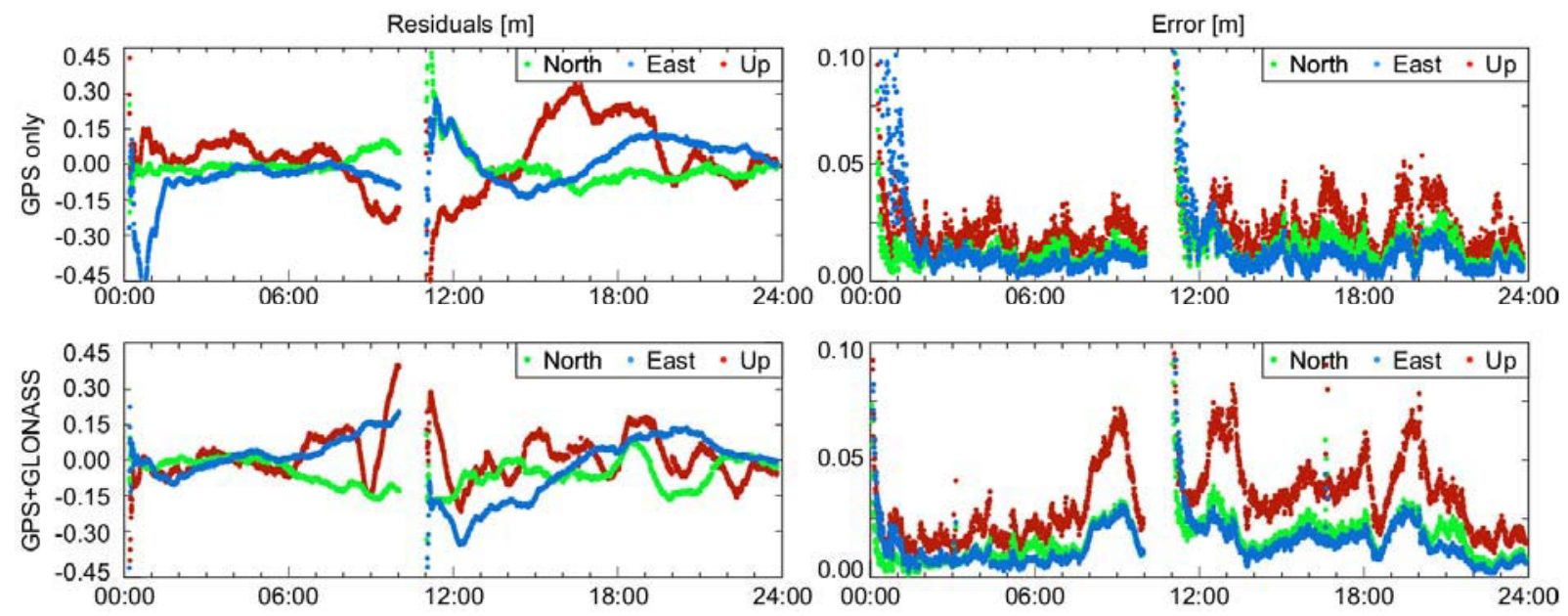

Fig. 6. Time series of residuals and estimated errors for GPS only with IGS02 stream (top) and GPS+GLONASS with IGS03 stream (bottom) real-time positioning in kinematic mode for station WROC, DOY 114, 2014 
The representative results of real-time GPS only and GPS+GLONASS processing are presented in Figure 5 for static mode and Figure 6 for kinematic mode. A 1-hour gap from 10:00 to 11:00 was caused by a problem with stream reception by BNC software. The implemented cycle slip detectors recognized the gap, and the ambiguities were reset. In kinematic mode, the solution converged again, and in static mode it was possible to resolve float ambiguities immediately, as the coordinates were assumed to be know from previous epoch. In figure 6 it can be clearly seen that the GPS+GLONASS static solution was much less accurate than GPS only solution. The estimated errors, however, were very similar in both solutions meaning that the quality was overestimated in the GPS+GLONASS solution. From the results of the kinematic positioning one can see the shorter convergence time for the GPS+GLONASS solution. In this case, the coordinate residuals were below 1 decimeter level just after few epoch of processing.

\section{TROPOSPHERE CONSTRAINING}

A common procedure in PPP is to have the adjustment model account for the correction of an a priori value of the troposphere delay given at the first epoch of data processing, and have the delay filter updated epoch by epoch. This approach requires some time so that a change in constellation geometry allows to efficiently de-correlate among tropospheric delay, receiver clock error, and height. Empirical tropospheric state models and mapping functions are available, however, they may not properly reflect the actual state of the troposphere, especially in severe weather conditions. In this case, it would be more appropriate to make use of a regional troposphere model based on real-time or near-real time (NRT) measurements. In this context, it may be effective to constrain the tropospheric delay using external information coming from NRT analysis of the regional GBAS network (Wielgosz et al. 2011, Hadas et al. 2013, Kalita et al. 2014).

To evaluate the difference between the unconstrained and constrained solutions, 7 days of 0.1 $\mathrm{Hz}$ GPS data from selected Polish EPN stations were processed simultaneously in two approaches. The first one was a standard kinematic PPP procedure using VMF to take advantage from low elevation GNSS satellites. VMF was also used as an a priori troposphere model. IGS RTS products from IGS03 stream were used. In the second approach, the NRT ZTD model developed at WUELS (Bosy et al 2010, Bosy et al 2012) was used to constrain the troposphere delay correction estimates, by including the additional equation into a functional model:

$$
\delta Z T D=Z T D^{N R T}-Z T D^{\prime}
$$

where

- $Z T D^{N R T}$ is the zenith troposphere delay from near-real time regional model;

- $Z T D^{\prime}$ is the a priori zenith troposphere delay value;

- $\delta Z T D$ is the correction to the a priori zenith troposphere delay value.

The standard deviation for the additional equation was set to $10 \mathrm{~mm}$, since the quality of applied NRT ZTD model estimated with respect to the EPN final combined troposphere product and ASG-EUPOS rapid solutions is $8 \mathrm{~mm}$ of standard deviation of ZTD differences with average bias of $-1.8 \mathrm{~mm}$. The other settings in the second approach were the same as in the first approach. The selected period of experimentation was characterized by changing weather conditions that influenced the troposphere delay (see Figure 7). 
The exemplary results for unconstrained solution are presented in Figure 8, left. North and East residuals are below $10 \mathrm{~cm}$ after the convergence time, which is usually between 1 to 2 hours. The Up component is not as well determined, sensitive to the number of satellites, and strongly correlates with estimated troposphere delay. In extreme cases, the residuals are larger than $0.7 \mathrm{~m}$, and the estimated error exceeds $20 \mathrm{~cm}$. Additionally, some cases were observed when the quality of the solution suddenly got slightly worse for all stations simultaneously, which is probably related to the poor quality of RTS products. During the processing period, the estimated troposphere delay differs up to $12 \mathrm{~cm}$ from reference NRT ZTD solution. Although the interval of estimated and reference ZTD are different, such big residuals and fluctuations in real time estimated troposphere delay seems to be unrealistic. At the same time, the largest residuals and errors in the Up coordinate occurs, when the estimated ZTD differs the most from the regional ZTD model.

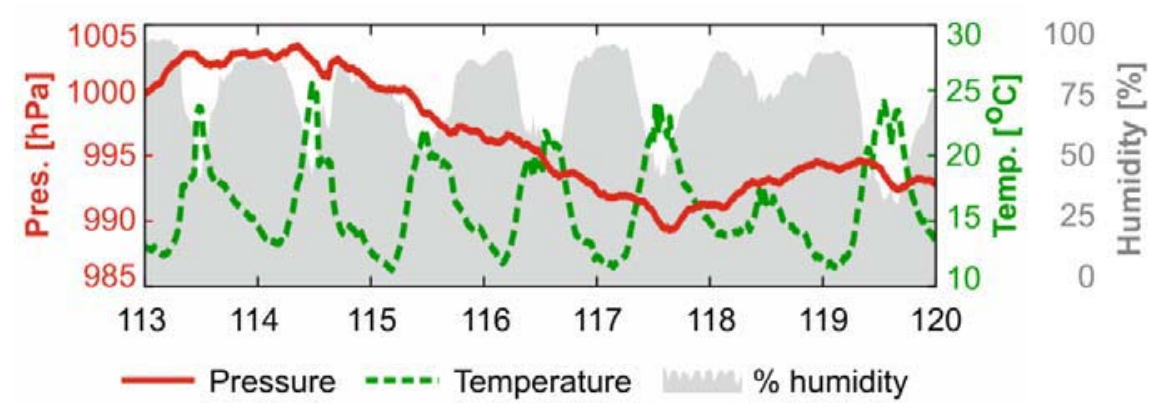

Fig. 7. Values of atmospheric parameters during the time of experiment at station WROC, DOY $113-119,2014$
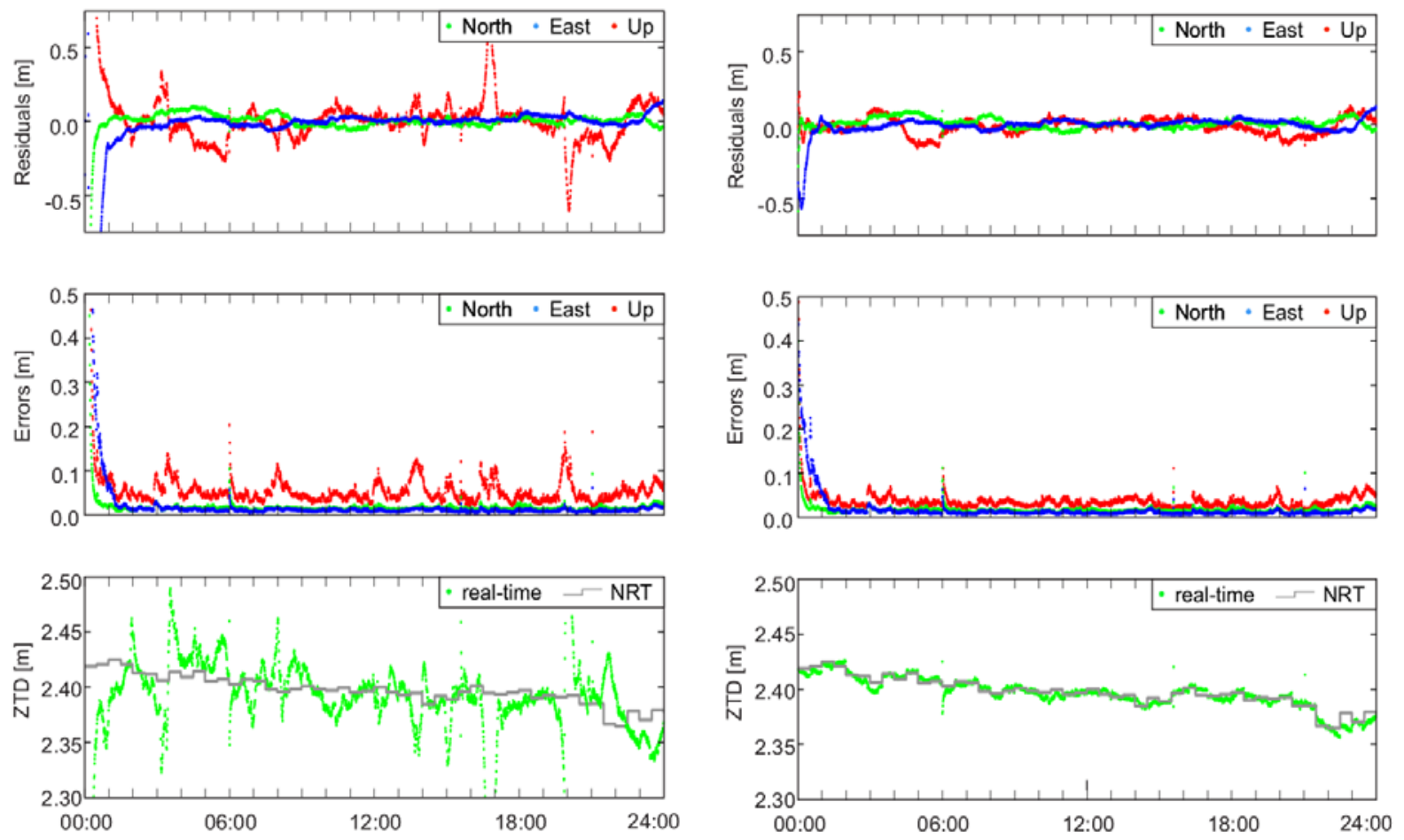

Fig. 8. Results of unconstrained (left) and NRT-ZTD constrained (right) kinematic PPP solution for station WROC, DOY 116, 2014 
In case the troposphere delay is constrained (Figure 8, right), all three coordinates are accurate and precise, although the error of the Up component ( $5 \mathrm{~cm}$ on average) is still slightly larger than the error of the East and North components $(2 \mathrm{~cm}$ on average). After the solution has converged, the height residuals do not exceed $20 \mathrm{~cm}$, and are smaller than $15 \mathrm{~cm}$ for $91 \%$ of time. From the very beginning of the data processing, the residuals for all three coordinates are much smaller, even though the estimated error is relatively large. The common cases of sudden degradation of the solution are still present.

After processing the 7-day long data from 10 Polish EPN stations in PPP kinematic mode in unconstrained and constrained approaches, the mean bias and standard deviation of coordinate residuals, with respect to known EPN coordinates, were calculated (Figure 9). In both approaches the results for the North and East components are very similar. The NRT-ZTD model shifts the height solution by about $1 \mathrm{~cm}$ and at the same time stabilize the solution over time. The standard deviation for all stations is reduced on average by $40 \%$, from $14 \mathrm{~cm}$ to $8 \mathrm{~cm}$. The results confirm the usefulness of near-real time troposphere delay models in real-time PPP kinematic processing and a significant improvement should be noticed in unusual or severe weather conditions.
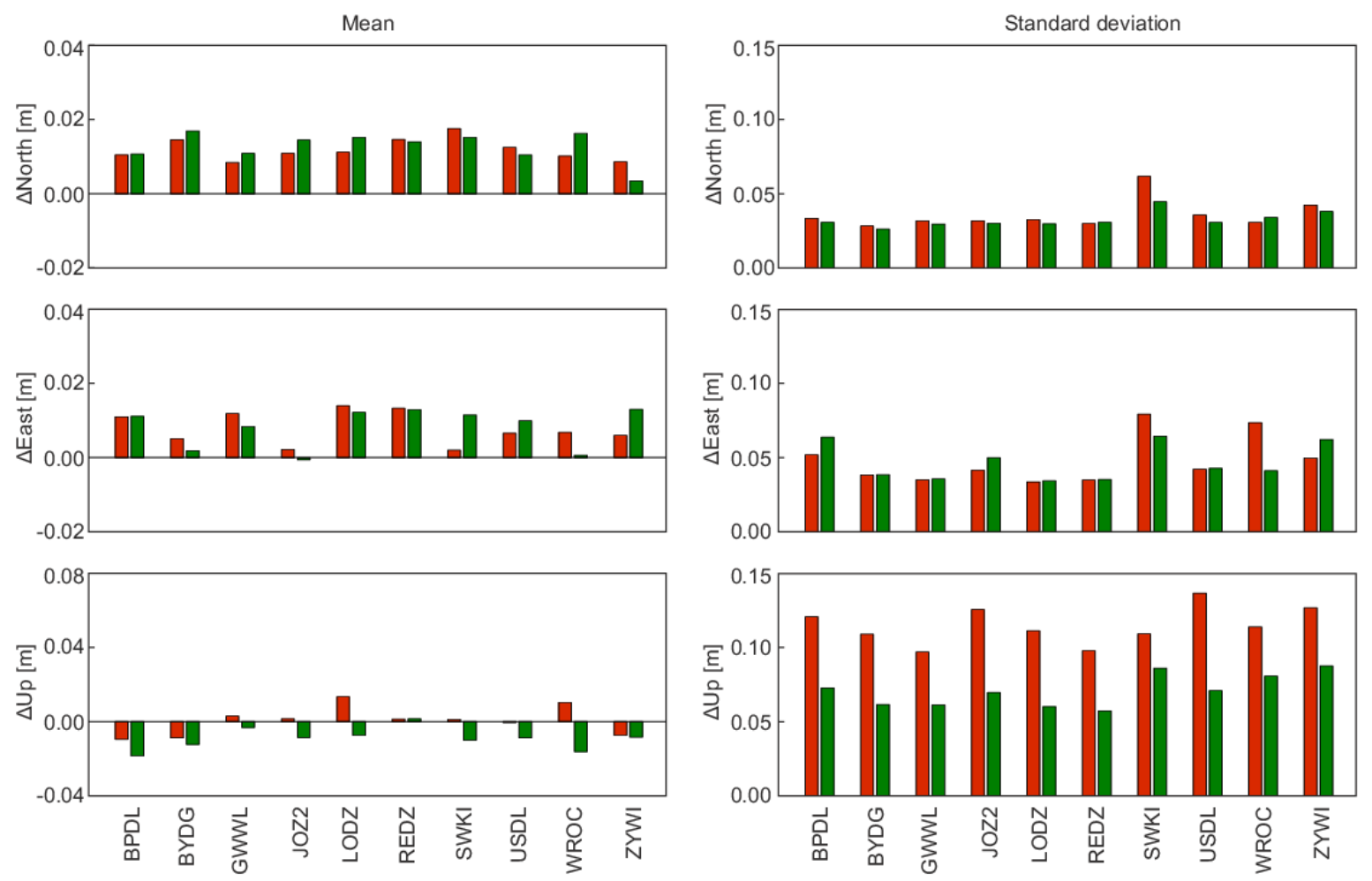

Fig. 9. Statistics of residuals for processed EPN stations over DOY 113-119, 2014, using unconstrained (red) and NRT-ZTD constrained (green) approach.

\section{REAL-TIME TROPOSPHERE ESTIMATION}

Although the software was originally developed for position determination, it can also be used to estimate other parameters. In particular, the GNSS-WARP can provide real-time troposphere delay estimates, as a by-product of static positioning. The growing importance of near real-time ZTD estimation for numerical weather prediction models results in some first efforts on real-time ZTD estimation. To evaluate the quality of real-time troposphere delay estimates with GNSS- 
WARP, a time-limited postprocessing benchmark campaign was analyzed. One week of daily GPS data from 5 Polish EPN stations was processed in static mode using VMF mapping functions for satellites with elevation of 5 degrees or higher. Selected stations were distributed evenly across the country and represent different elevations and geographical conditions. Due to the limited software functionality in post-processing mode, the ZTD were estimated every 30 seconds and the solution was reinitialized every night at midnight. Such limitations will not occur for the real-time running software.

The exemplary time series of ZTD and ZTD error estimated in real-time is presented in Figure 10. ZTD from EPN final solution were considered as a reference. In general, the real-time ZTD followed the trend of ZTD changes. The mean error of estimated ZTD was $9.0 \mathrm{~mm}$. The mean value of residuals was $-2.5 \mathrm{~mm}$ with standard deviation of $11.2 \mathrm{~mm}$. The residuals reached up to $6 \mathrm{~cm}$ in extreme cases. In this instance is important to note, however, that the EPN results represent the mean value of ZTD over a 1-hour period, while the real-time estimates are instantaneous.

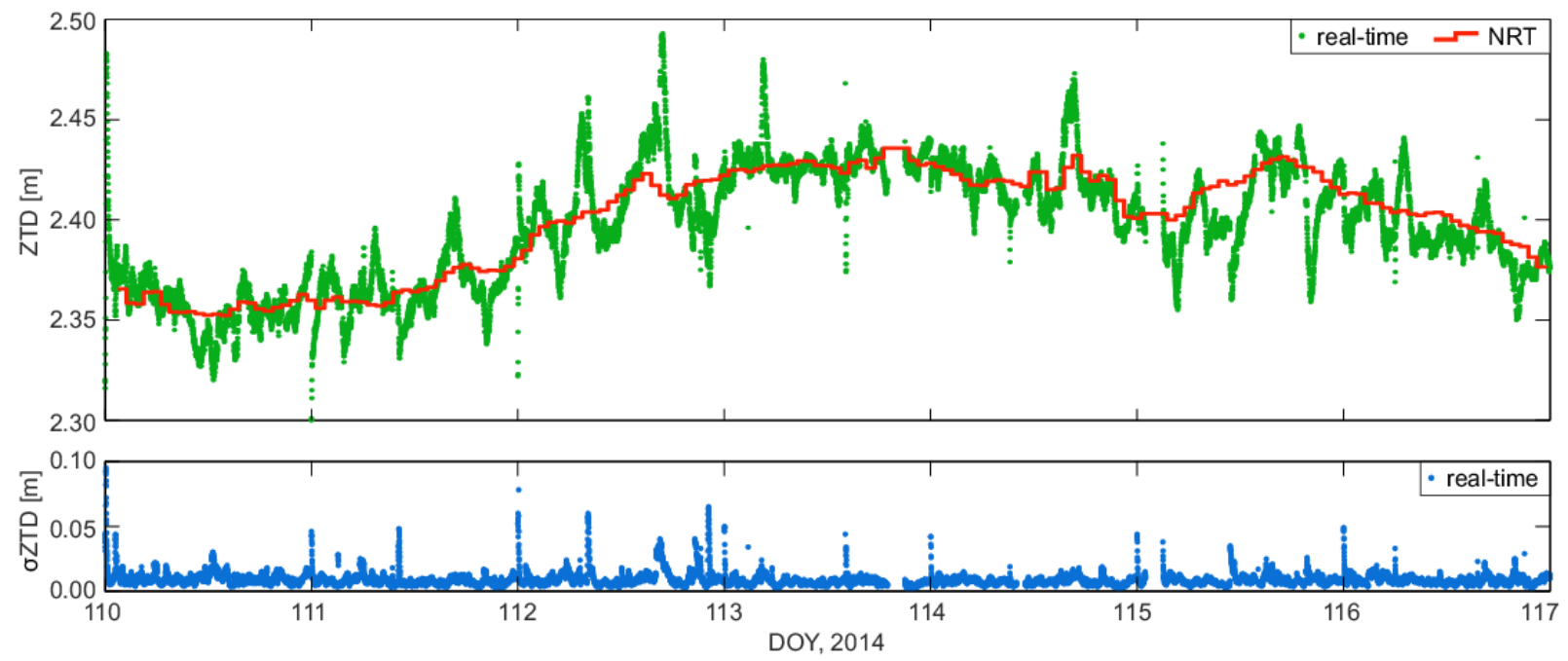

Fig. 10. Real-time ZTD (green) and its error (blue) compared with EPN final ZTD (red) at station WROC over DOY 110-117, 2014

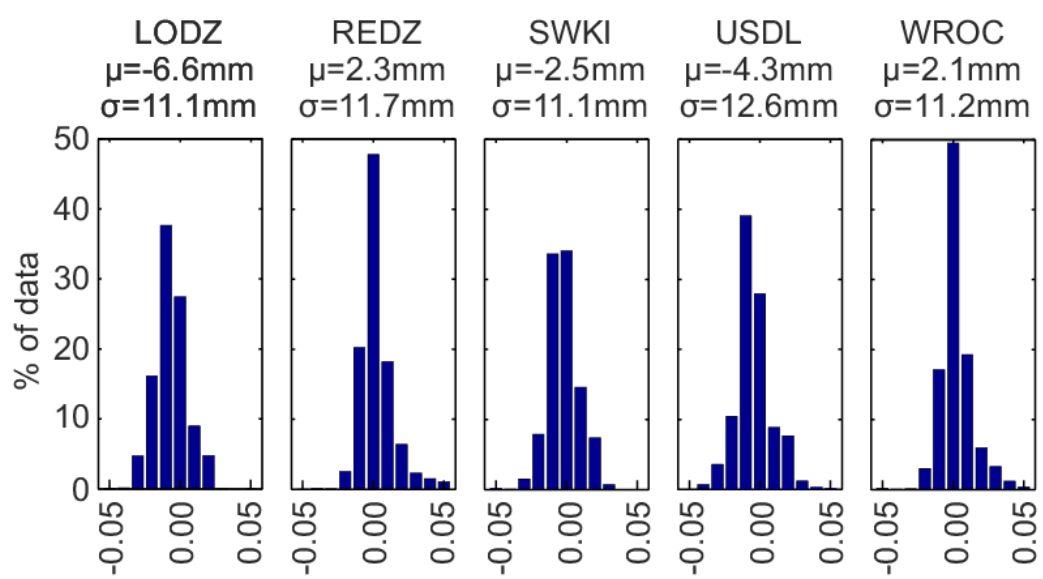

Fig. 11. Histograms of differences between real-time estimated ZTD and EPN final solution over selected Polish EPN stations over DOY 110-117, 2014 
The normalized histograms of all residuals for each station processed are presented in Figure 11. Presented histograms are similar to each other and the residual distributions are close to a normal distribution. For each station the standard deviation of residuals was at about $1 \mathrm{~cm}$. The real-time results were shifted by a few millimeters with respect to the EPN solution. More stations and weeks need to be processed to determine whether the shift is station-specific or not.

The quality of real-time ZTD obtained with GNSS-WARP is similar to the one presented in (Dousa and Vaclavovic, 2014). Still, the accuracy of the results is not enough for their assimilation into numerical weather prediction models, where $6 \mathrm{~mm}$ ZTD accuracy is required. The advantage of the presented solution is the low-latency and high-temporal resolution of derived ZTD, which is far better than the required $5 \mathrm{~min}$ repetition cycle and $5 \mathrm{~min}$ for latency. Further studies on averaging the solution over time should be performed to find the compromise between the accuracy, frequency, and latency of the results that will fulfill the target requirements.

\section{CONCLUSIONS}

In order to challenge PPP limitation, the GNSS-WARP (Wroclaw Algorithms for Real-time Positioning) has been developed from scratch at Wroclaw University of Environmental and Life Science (WUELS), Poland. In order to confirm the proper implementation of GNSS data handling and adjustment model, numerical testing with IGS final products were performed in static and kinematic modes. The analysis of residuals from the official IGb08 of processing stations allowed us to consider the GNSS-WARP as state-of-the-art software and to continue further studies on PPP algorithms development.

Eventually, the software was used for real-time positioning using IGS real-time products. Tests carried out in static mode confirm, that the quality of GLONASS products is not yet as high as for GPS products. When only GPS products were used, a few millimeters of accuracy and precision were reached for all coordinate components in static mode, while the inclusion of GLONASS led to centimeter-level accuracy results. In kinematic mode, the use of GLONASS data led to improved determination of point height, while the horizontal coordinates were as well determined as in GPS only mode.

The developed software was also used to assess the impact of troposphere model application in positioning models. By constraining the real-time kinematic solution with near real-time regional troposphere model, the standard deviation of height residuals were reduced by $40 \%$. From the very beginning of the data processing, the residuals for all three coordinates are at a decimeter level, which is a promising result for further studies on fast ambiguity fixing in PPP.

Finally, the GNSS-WARP was used to estimate zenith troposphere delay in real-time mode. The general trend of real-time ZTD and EPN final estimates was similar. In some epoch, however, the differences reached up to $6 \mathrm{~cm}$. The mean value of residuals was $-2.5 \mathrm{~mm}$ with standard deviation of $11.2 \mathrm{~mm}$, so further improvements are required before the results can be useful for numerical weather prediction models.

Further developments of the software will concentrate on importing ionosphere maps for raw data processing, ambiguity techniques implementation, and development of real-time processing algorithms resistant to IGS RTS products outliers. 
Acknowledgments. This work is co-financed by the European Union as part of the European Social Fund, supported by the Ministry of Science and Higher Education Research Project No 2012/07/N/ST10/03716 and EU COST Action ES1206. Matlab Software License No.: 101979 provided by Wroclaw Center of Networking and Supercomputing (http: www.wcss.wroc.pl) computational grant was used for computations. The author gratefully acknowledge IGS for providing real-time streams and Bundesamt für Kartographie und Geodäsie (BKG) for providing the open-source BNC software. Acknowledgment goes to Marcelo Santos for sharing his knowledge on PPP algorithms and experience in software development.

\section{REFERENCES}

BKG (2013). BKG Ntrip Client (BNC) Version 2.8 manual, Federal Agency for Cartography and Geodesy, Frankfurt, Germany

Böhm, J., Kouba, J., and Schuh, H. (2009). Forecast Vienna Mapping Functions 1 for real-time analysis of space geodetic observations, Journal of Geodesy, 86(5), 397-401.

Bosy, J., Kaplon, J., Rohm, W., Sierny, J., and Hadas T. (2012). Near real-time estimation of water vapour in the troposphere using ground GNSS and the meteorological data, Annales Geophysicae, 30, 1379-1391.

Bosy, J., Rohm, W., and Sierny, J. (2010). The concept of Near Real Time atmosphere model based of GNSS and meteorological data from ASG-EUPOS reference stations, Acta Geodynamica et Geomaterialia, 7(3), 253-261.

Chen J., Ge M., Dousa J., and Gendt G. (2009). Evaluation of EPOS-RT for Real-time Deformation Monitoring, Journal of Global Positioning Systems, 8(1), 1-5.

Dousa, J., and Vaclavovic, P. (2014). Real-time zenith tropospheric delays in support of numerical weather prediction applications, Advances in Space Research, 53, 1347-1358.

Dow, J. M., Neilan, R. E., and Rizos, C. (2009). The international GNSS Service in a changing landscape of global navigation satellite systems, Journal of Geodesy, 83, 191-198.

Geng, J., Teferle, F. N., Meng, X., and Dodson, A. H. (2010). Towards PPP-RTK: ambiguity resolution in real-time precise point positioning, Advances in Space Research, 47, 1664-1673.

Hadas T., and Bosy J. (2014). IGS RTS precise orbits and clocks verification and quality degradation over time, GPS Solutions, 19(1), 93-105

Hadas, T., Kaplon, J., Bosy, J., Sierny, J., and Wilgan, K. (2013). Near-real-time regional troposphere models for the GNSS precise point positioning technique, Measurement Science and Technology, 24(2013), 055003 (12pp).

Hefty J., and Gerhatova L. (2012). Potential of Precise Point Positioning using 1 Hz GPS data for detection of seismic-related displacements. Acta Geodynamica et Geomaterialia, 9(3), 303313.

Héroux, P., Kouba, J., Collins, P., and Lahaye, F. (2000). GPS carrier-phase point positioning with precise orbit products, Proceedings of International Symposium on Kinematic Systems in Geodesy, Geomatics and Navigation 2001, Banff, Alberta, Canada.

Jensen, A. B. O., and Ovstedal, O. (2008). The effect of different tropospheric models on precise point positioning in kinematic mode, Survey Review, 40(308), 173-187. 
Juan, J. M., Hernandez-Pajares, M., Sanz, J., Ramos-Bosch, P., Aragon-Angel, A., Orus, R., Ochieng, W., Feng, S., Jofre, M., Coutinho, P., Samson, J., and Tossaint, M., (2012). Enhanced Precise Point Positioning for GNSS Users, IEEE Transactions on Geoscience and Remote Sensing, 50(10), 4213-4222.

Kalita J.Z., Rzepecka Z., and Krzan G., (2014). Evaluation of the possibility of using the predicted tropospheric delays in real time GNSS positioning, Artificial Satellites, 49(4), 179189

Kanzaki M, Matsushita Y., Kakimoto H., Rocken C., Iwabuchi T., Mervart L., Johnson J., and Lukes Z. (2011). GNSS Positioning of Ocean Buoys in Japan for Disaster Prevention, Proceedings of 24th International Technical Meeting of The Satellite Division of the Institute of Navigation, Institute of Navigation, Portland, OR, 717-723.

Kouba, J. (2009). A guide to using International GNSS Service (IGS) products, Geodetic Survey Division, Natural Resources Canada, http://igscb.jpl.nasa.gov/igscb/resource/pubs/UsingIGSProductsVer21.pdf

Lagler, K., Schindelegger, M., Böhm, J., Krásná, H., and Nilsson, T. (2013). GPT2: Empirical slant delay model for radio space geodetic techniques, Geophysical Research Letters, 40(6), $1069-1073$

Laurichesse, D. (2011). The CNES Real-time PPP with Undifferenced Integer Ambiguity Resolution Demonstrator, Proceedings of 24th International Technical Meeting of The Satellite Division of the Institute of Navigation, Institute of Navigation, Portland, OR, 654662.

Leandro, R. F., Santos, M. C., and Langley, R. B. (2006). UNB neutral atmosphere models: development and performance, Proceedings of 2006 National Technical Meeting of The Institute of Navigation, Monterey, CA, 564-573.

Leandro, R. F., Santos, M. C., and Langley, R. B. (2007). GAPS: The GPS Analysis and Positioning Software - A Brief Overview, Proceedings of 20th International Technical Meeting of The Satellite Division of the Institute of Navigation, Institute of Navigation, Fort Worth, TX, 1807-1811.

Li, X., Zhang, X., and Ge, M. (2011). Regional reference network augmented precise point positioning for instantaneous ambiguity resolution, Journal of Geodesy, 85, 151-158.

Mervart, L., and Weber, G. (2011). Real-time combination of GNSS orbit and clock correction streams using a Kalman filter approach, Proceedings of 24th International Technical Meeting of The Satellite Division of the Institute of Navigation, Institute of Navigation, Portland, OR, $707-711$.

Weber, G., Dettmering, D., and Gebhard, H. (2005). Networked transport of RTCM via Internet Protocol (NTRIP), IAG Symposium, 128, 60-64.

Wielgosz P., Paziewski J., and Baryla R. (2011). On Constraining Zenith Tropospheric Delays in Processing of Local GPS Networks with Bernese Software, Survey Review, 43(323), 472-483

Wübbena, G., Schmitz, M., and Bagge, A. (2005). PPP-RTK: precise point positioning using state-space representation in RTK networks, Proceedings of 24th International Technical Meeting of The Satellite Division of the Institute of Navigation, Institute of Navigation, Long Beach, CA, 2584-2594. 
Zhang, H., Gao, Z., Ge, M., Niu, X., Huang, L., Tu, R., and Li, X. (2013). On the Convergence of Ionospheric Constrained Precise Point Positioning (IC-PPP) Based on Undifferential Uncombined Raw GNSS Observations, Sensors (Basel), 13(11): 15708-15725.

Zumberge, J. F., Heflin, M. B., Jefferson, D. C., Watkins, M. M., and Webb, F. H. (1997). Precise point positioning for the efficient and robust analysis of GPS data from large networks, Journal of Geophysical Research, 102, 5005-5018.

Received: 2015-02-13,

Reviewed: 2015-02-26,

Accepted: 2015-03-01. 